\title{
Semantics for Active Integrity Constraints Using Approximation Fixpoint Theory*
}

\author{
Bart Bogaerts $^{\dagger}$ and Luís Cruz-Filipe ${ }^{\ddagger}$ \\ $\dagger$ KU Leuven, Department of Computer Science \\ Celestijnenlaan 200A, Leuven, Belgium \\ $\ddagger$ University of Southern Denmark, Department of Mathematics and Computer Science \\ Campusvej 55, Odense, Denmark \\ bart.bogaerts@cs.kuleuven.be, lcfilipe@gmail.com
}

\begin{abstract}
Active integrity constraints (AICs) constitute a formalism to associate with a database not just the constraints it should adhere to, but also how to fix the database in case one or more of these constraints are violated. The intuitions regarding which repairs are "good" given such a description are closely related to intuitions that live in various areas of non-monotonic reasoning.

In this paper, we apply approximation fixpoint theory, an algebraic framework that unifies semantics of non-monotonic logics, to the field of AICs. This results in a new family of semantics for AICs, of which we study semantics and relationships to existing semantics. We argue that the AFT-wellfounded semantics has some desirable properties.
\end{abstract}

\section{Introduction}

One of the key components of modern-day databases are integrity constraints: logical formulas that specify semantic relationships between the data being modeled that have to be satisfied at all times. When the database is changed (typically by updating), it is necessary to check if its integrity constraints still hold; in the negative case, it must be repaired.

Database repairs have been an important topic of research for many years [Abiteboul, 1988]. There are two major problems when deciding how to repair an inconsistent database: finding possible repairs and choosing which one to apply. Indeed, there are typically several ways to fix an inconsistent database, and several criteria to choose the "best" one have been proposed over the years. Among the most widely accepted criteria are minimality of change [Winslett, 1990; Eiter and Gottlob, 1992] - change as little as possible - and the common-sense law of inertia [Przymusinski and Turner, 1997] - do not change anything without a reason to do it.

A typical implementation of integrity constraints in database systems is by means of event-condition-action rules [Teniente and Olivé, 1995; Widom and Ceri, 1996],

\footnotetext{
${ }^{*}$ Bart Bogaerts is a postdoctoral fellow of the Research Foundation - Flanders (FWO). Luís Cruz-Filipe was partially supported by the Danish Council for Independent Research, Natural Sciences, grant DFF-1323-00247.
}

which specify update actions to be performed when a particular event (a trigger) occurs and specific conditions hold. However, these rules do not have a declarative semantics, making their joint behavior hard to understand.

The formalism of active integrity constraints (AICs) [Flesca et al., 2004] was inspired by a similar idea. AICs express database dependencies through logic programmingstyle rules that include update actions in their heads. They come with a set of declarative semantics [Caroprese and Truszczyński, 2011] that identifies several progressively more restricted classes of repairs, which can be used as criteria to select a preferred repair. These repairs can be computed by means of tree algorithms[Cruz-Filipe et al., 2013; Cruz-Filipe et al., 2015].

It is striking that many intuitions about "good" repairs, such as minimality of change, are similar to intuitions that surfaced in other domains of non-monotonic reasoning, such as logic programming [van Emden and Kowalski, 1976] and default logic [Reiter, 1980]. Still, it has been hard to find satisfying semantics for AICs. As shown by Cruz-Filipe et al. [2013], the semantics of founded repairs [Caroprese et al., 2006] unexpectedly fails to respect the common-sense law of inertia, while the more restricted semantics of justified repairs [Caroprese and Truszczyński, 2011] forbids natural repairs. That work proposed the operational semantics of wellfounded repairs, which however is not modular [Cruz-Filipe, 2014] and therefore restricted in its practical applicability.

In this paper, we define a new class of semantics for AICs that are natural counterparts of existing semantics in various non-monotonic reasoning domains. To be precise, we define semantics for AICs based on approximation fixpoint theory $(A F T)$ [Denecker et al., 2000], an abstract algebraic framework that unifies semantics of logic programming, default logic, autoepistemic logic [Moore, 1985], abstract argumentation frameworks [Dung, 1995] and abstract dialectical frameworks [Brewka and Woltran, 2010], as shown by Denecker, Marek and Truszczyński [2000; 2003] and Strass [2013]. In order to do so, we continue the work of Cruz-Filipe [2016], who defined a semantic operator for AICs and used it to study the grounded fixpoint semantics [Bogaerts et al., 2015a] of AICs. In this paper, we define an approximating operator of Cruz-Filipe's semantic operator. From this operator, approximation fixpoint theory immediately provides us with (i) a supported fixpoint semantics, (ii) a well-founded 
fixpoint semantics, (iii) a (partial) stable fixpoint semantics, (iv) a Kripke-Kleene fixpoint semantics, and (v) a (partial) grounded fixpoint semantics. We study properties of these new semantics and study how they compare to existing semantics. We argue that the AFT-style well-founded semantics is valuable. Indeed, we show that this semantics can be computed in polynomial time, and that, on practical examples, it corresponds to the intuitions underlying database repairs, providing natural upper and lower bounds on the set of acceptable repairs (formally: the AFT-style well-founded model approximates all justified, stable and grounded repairs).

Besides defining a new class of interesting semantics, our work provides solid foundations for transferring work from the non-monotonic reasoning domain to AICs. For instance, we can now directly apply existing results from AFT, such as modularity results [Vennekens et al., 2006; Bogaerts et al., 2016] or predicate introduction results [Vennekens et al., 2007a; 2007b]. Following Caroprese and Truszczyński [2011], our work also paves the way to applying AFT to revision programming.

\section{Preliminaries}

Active Integrity Constraints We assume a fixed set $A t$ of atoms. An interpretation or database is a subset of At. A literal is an atom $a$ or its negation $\neg a$. We say $\neg a$ and $a$ are dual literals and denote the dual of a literal $l$ by $l^{D}$. The satisfaction relation between databases $D B$ and literals is defined as usual: $D B=a$ if $a \in D B$ and $D B \models \neg a$ if $D B \not \models a$.

An update action has the form $+a$ or $-a$ with $a \in A t$. We call $+a$ and $-a$ dual actions and use $\alpha^{D}$ to denote the dual action of $\alpha$. Update actions represent changes to the database: $+a$ transforms $D B$ to $D B \cup\{a\}$ and $-a$ transforms $D B$ to $D B \backslash\{a\}$. A set of update actions $\mathcal{U}$ is consistent if it does not contain an action and its dual. A consistent set of update actions $\mathcal{U}$ acts on a database $D B$ by executing all its actions simultaneously; we denote the result of this operation by $\mathcal{U}(D B)$. Following Cruz-Filipe [2016], we define an operation $\biguplus$ on consistent sets of update actions:

$\mathcal{U}_{1} \biguplus \mathcal{U}_{2}=\left(\mathcal{U}_{1} \cup\left\{\alpha \in \mathcal{U}_{2} \mid \alpha^{D} \notin \mathcal{U}_{1}\right\}\right) \backslash\left\{\alpha \in \mathcal{U}_{1} \mid \alpha^{D} \in \mathcal{U}_{2}\right\}$

This operation has the property that: if every action in $\mathcal{U}_{1}$ changes $D B$ and every action in $\mathcal{U}_{2}$ changes $\mathcal{U}_{1}(D B)$, then

$$
\left(\mathcal{U}_{1} \biguplus \mathcal{U}_{2}\right)(D B)=\mathcal{U}_{2}\left(\mathcal{U}_{1}(D B)\right) \text {. }
$$

Literals and update actions are related by mappings lit and ua, where lit $(+a)=a$, lit $(-a)=\neg a$ and ua is the inverse of lit. These mappings naturally extend to sets of literals/actions.

Definition 2.1. An active integrity constraint (AIC) is a rule $r$ of the form

$$
l_{1} \wedge \cdots \wedge l_{n} \supset \alpha_{1}|\cdots| \alpha_{k}
$$

such that $\operatorname{lit}\left(\alpha_{i}^{D}\right) \in\left\{l_{1}, \ldots, l_{n}\right\}$ for each $i$. We call $l_{1} \wedge \cdots \wedge$ $l_{n}$ the body of $r$, denoted body $(r)$, and $\alpha_{1}|\cdots| \alpha_{k}$ the head of $r$, denoted head $(r)$.

The informal reading of the above rule is: "If each of the $l_{i}$ holds in $D B$, then $D B$ is inconsistent. An allowed fix is to execute one or more of the $\alpha_{i} . "$ A set of AICs represents constraints a database should adhere to and, in addition, which atoms can be changed in order to fix it. Intuitively, atoms can only be changed if there is some rule that allows it.

An AIC is normal if $k=1$. The normalization of an AIC of the form (1) is the set of AICs

$$
\left\{l_{1} \wedge \cdots \wedge l_{n} \supset \alpha_{i} \mid 1 \leq i \leq k\right\} .
$$

It follows from the informal explanation above that we expect normalization to preserve semantics. This is the case for most semantics of AICs (the notorious exception being the semantics of justified repairs [Caroprese and Truszczyński, 2011], which poses several other problems [Cruz-Filipe et al., 2013]). In the current paper, we assume all AICs are normal. Extensions of the semantics we define for non-normal AICs can then be obtained through normalization, if needed.

Definition 2.2. A set of update actions $\mathcal{U}$ is a weak repair for $D B$ and a set $\eta$ of AICs (shortly, for $\langle D B, \eta\rangle$ ) if:

- every action in $\mathcal{U}$ changes $D B$, and

- $\mathcal{U}(D B) \forall \operatorname{body}(r)$ for each $r \in \eta$.

$\mathrm{A} \subseteq$-minimal weak repair is called a repair.

(Weak) repairs do not take the head of AICs into account, and thus allow arbitrary changes to the database. We now review semantics for AICs that have been defined with the intention to allow only changes allowed by one of the AICs.

Definition 2.3 ([Caroprese et al., 2006]). A set of update actions $\mathcal{U}$ is founded with respect to $\langle D B, \eta\rangle$ if, for each $\alpha \in \mathcal{U}$, there is a rule $r \in \eta$ with $\alpha \in \operatorname{head}(r)$ and such that $\mathcal{U}^{\prime}(D B) \models \operatorname{body}(r)$, where $\mathcal{U}^{\prime}=\mathcal{U} \backslash\{\alpha\}$. A founded (weak) repair is a (weak) repair that is founded.

Definition 2.4 ([Cruz-Filipe et al., 2013]). A (weak) repair $\mathcal{U}$ for $\langle D B, \eta\rangle$ is well-founded if there exists a sequence of actions $\alpha_{1}, \ldots, \alpha_{n}$ such that $\mathcal{U}=\left\{\alpha_{1}, \ldots, \alpha_{n}\right\}$ and, for each $i \in\{1, \ldots, n\}$, there is a rule $r_{i}$ such that $U_{i-1}(D B) \models$ body $\left(r_{i}\right)$ and $\alpha_{i} \in \operatorname{head}\left(r_{i}\right)$, where $U_{i-1}=\left\{\alpha_{1}, \ldots, \alpha_{i-1}\right\}$.

Definition 2.5 ([Caroprese and Truszczyński, 2011]). Let $\mathcal{U}$ be a set of update actions and $\langle D B, \eta\rangle$ a database.

- The no-effect actions with respect to $D B$ and $\mathcal{U}$, neff $_{D B}(\mathcal{U})$, are the actions that change neither $D B$, nor $\mathcal{U}(D B)$.

- The set of non-updatable literals of an AIC $r, \operatorname{nup}(r)$, contains all body literals of $r$ that do not occur in the head of $r$.

- $\mathcal{U}$ is closed under $\eta$ if for each $r \in \eta$, ua $(\operatorname{nup}(r)) \subseteq \mathcal{U}$ implies $\operatorname{head}(r) \cap \mathcal{U} \neq \emptyset$.

- $\mathcal{U}$ is a justified action set if it is a minimal superset of neff $_{D B}(\mathcal{U})$ closed under $\eta$.

- $\mathcal{U}$ is a justified (weak) repair if it is a (weak) repair and $\mathcal{U} \cup$ neff $_{D B}(\mathcal{U})$ is a justified action set.

Although the notion of closed set of actions does not take the database into account, its role in the definition of justified weak repairs is as part of the definition of justified action setwhere all actions that do not change the database are included.

Marek and Truszczynski [1998] defined in the context of revision programming the shifting property; this property was later transferred to active integrity constraints [Caroprese and 
Truszczyński, 2011]. Intuitively, a semantics for AICs possesses the shifting property if uniformly replacing some literals with their duals preserves the semantics at hand.

Definition 2.6. Let $S \subseteq A t$ be a set of atoms and $l$ a literal. The shift of $l$ with respect to $S$ is defined as

$$
\operatorname{shift}_{S}(l)= \begin{cases}l & \text { if } l \notin S \\ l^{D} & \text { otherwise }\end{cases}
$$

The shift function is extended to sets of literals, update actions and AICs in the straightforward manner.

Definition 2.7. We say that a semantics for AICs has the shifting property if: for all $\langle D B, \eta\rangle$ and all $S \subseteq A t, \mathcal{U}$ is a repair of $\langle D B, \eta\rangle$ accepted by the semantics if and only if shift $_{S}(\mathcal{U})$ is a repair of $\left\langle\operatorname{shift}_{S}(D B)\right.$, shift $\left.{ }_{S}(\eta)\right\rangle$ accepted by the semantics.

If a semantics has the shifting property, then we can reduce any situation to the case $D B=\emptyset$ by taking $S=D B$.

Lattices, Operators and Fixpoints A partially ordered set (poset) $\langle L, \leq\rangle$ is a set $L$ equipped with a partial order $\leq$, i.e., a reflexive, antisymmetric, transitive relation. As usual, we write $x<y$ as abbreviation for $x \leq y \wedge x \neq y$. If $S$ is a subset of $L$, then $x$ is an upper bound, respectively a lower bound of $S$ if for every $s \in S$, it holds that $s \leq x$, respectively $x \leq s$. An element $x$ is a least upper bound (respectively greatest lower bound of $S$ ) if it is an upper bound that is smaller than every other upper bound (resp. a lower bound that is greater than every other lower bound). If $S$ has a least upper bound (resp. a greatest lower bound) we denote it lub $(S)$ (resp. glb $(S))$. As is custom, we sometimes call a greatest lower bound a meet, and a least upper bound a join and use the related notations $\bigwedge S=\operatorname{glb}(S), x \wedge y=\operatorname{glb}(\{x, y\})$, $\bigvee S=\operatorname{lub}(S)$ and $x \vee y=\operatorname{lub}(\{x, y\})$. We call $\langle L, \leq\rangle$ a complete lattice if every subset of $L$ has a least upper bound and a greatest lower bound. A complete lattice has both a least element $\perp$ and a greatest element $T$.

Since we apply our results to (finite) databases, for the sake of simplicity we assume $L$ to be finite in this text. All presented results easily generalize to the infinite setting as well.

An operator $O: L \rightarrow L$ is monotone if $x \leq y$ implies that $O(x) \leq O(y)$. An element $x \in L$ is a fixpoint of $O$ $O(x)=x$. Every monotone operator $O$ in a complete lattice has a least fixpoint, denoted $\operatorname{lfp}(O)$, which is the limit (the least upper bound) of the increasing sequence $\left(x_{i}\right)_{i \in \mathbb{N}}$ defined by $x_{0}=\perp$ and $x_{i+1}=O\left(x_{i}\right)$.

Bogaerts et al. [2015a] called a point $x \in L$ grounded for $O$ if, for each $v \in L$ such that $O(v \wedge x) \leq v$, it holds that $x \leq v$. Later, they generalized this notion to partial grounded fixpoints [Bogaerts et al., 2015b]. They explained the intuition underlying these concepts under the assumption that the elements of $L$ are sets of "facts" of some kind and the $\leq$ relation is the subset relation between such sets: in this case, a point $x$ is grounded if it contains only facts that are sanctioned by the operator $O$, in the sense that if we remove them from $x$, then the operator will add at least one of them again.
Approximation Fixpoint Theory Given a lattice $L$, approximation fixpoint theory (AFT) [Denecker et al., 2000] uses the bilattice $L^{2}$. We define two projection functions for pairs as usual: $(x, y)_{1}=x$ and $(x, y)_{2}=y$. Pairs $(x, y) \in L^{2}$ are used to approximate elements in the interval $[x, y]=\{z \mid x \leq z \wedge z \leq y\}$. We call $(x, y) \in L^{2}$ consistent if $x \leq y$, that is, if $[x, y]$ is non-empty, and use $L^{c}$ to denote the set of consistent elements. Elements $(x, x) \in L^{c}$ are called exact; they constitute the embedding of $L$ in $L^{2}$. We sometimes abuse notation and use the tuple $(x, y)$ and the interval $[x, y]$ interchangeably. The precision ordering on $L^{2}$ is defined as $(x, y) \leq_{p}(u, v)$ if $x \leq u$ and $v \leq y$. In case $(u, v)$ is consistent, this means that $(x, y)$ approximates all elements approximated by $(u, v)$, or in other words that $[u, v] \subseteq[x, y]$. If $L$ is a complete lattice, then $\left\langle L^{2}, \leq_{p}\right\rangle$ is also a complete lattice.

AFT studies fixpoints of lattice operators $O: L \rightarrow L$ through operators approximating $O$. An operator $A: L^{2} \rightarrow$ $L^{2}$ is an approximator of $O$ if it is $\leq_{p}$-monotone, and has the property that $A(x, x)=(O(x), O(x))$ for all $x$. Approximators are internal in $L^{c}$ (i.e., map $L^{c}$ into $L^{c}$ ). As usual, we often restrict our attention to symmetric approximators: approximators $A$ such that, for all $x$ and $y, A(x, y)_{1}=$ $A(y, x)_{2}$.Denecker et al. [2004] showed that the consistent fixpoints of interest (supported, stable, well-founded) are uniquely determined by an approximator's restriction to $L^{c}$, hence, sometimes we only define approximators on $L^{c}$.

AFT studies fixpoints of $O$ using fixpoints of $A$.

- The A-Kripke-Kleene fixpoint is the $\leq_{p}$-least fixpoint of $A$, and it approximates all fixpoints of $O$.

- A partial A-stable fixpoint is a pair $(x, y)$ such that $x=\operatorname{lfp}\left(A(\cdot, y)_{1}\right)$ and $y=\operatorname{lfp}\left(A(x, \cdot)_{2}\right)$, where $A(\cdot, y)_{1}$ denotes the operator $L \rightarrow L: x \mapsto A(x, y)_{1}$ and analogously for $A(x, \cdot)_{2}$.

- The A-well-founded fixpoint is the least precise partial $A$-stable fixpoint.

- An $A$-stable fixpoint of $O$ is a fixpoint $x$ of $O$ such that $(x, x)$ is a partial $A$-stable fixpoint. This is equivalent to the condition that $x=\operatorname{lfp}\left(A(\cdot, x)_{1}\right)$.

The $A$-Kripke-Kleene fixpoint of $O$ can be constructed as the limit of any monotone induction of $A$. For the $A$-wellfounded fixpoint, a similar constructive characterization has been worked out by Denecker and Vennekens [2007]:

Definition 2.8. An A-refinement of $(x, y)$ is a pair $\left(x^{\prime}, y^{\prime}\right) \in$ $L^{2}$ satisfying one of the following two conditions:

(i) $(x, y) \leq_{p}\left(x^{\prime}, y^{\prime}\right) \leq_{p} A(x, y)$, or

(ii) $x^{\prime}=x$ and $A\left(x, y^{\prime}\right)_{2} \leq y^{\prime} \leq y$.

An $A$-refinement is strict if $(x, y) \neq\left(x^{\prime}, y^{\prime}\right)$.

We call the first type (i.) of refinements application refinements and the second type (ii.) unfoundedness refinements. If $\left(x^{\prime}, y^{\prime}\right)$ is an $A$-refinement of $(x, y)$ and $A$ is clear from the context, we often write $(x, y) \rightarrow\left(x^{\prime}, y^{\prime}\right)$.

Definition 2.9. A well-founded induction of $A$ is a sequence $\left(x_{i}, y_{i}\right)_{i \leq n}$ with $n \in \mathbb{N}$ such that

- $\left(x_{0}, y_{0}\right)=(\perp, \top)$;

- $\left(x_{i+1}, y_{i+1}\right)$ is an A-refinement of $\left(x_{i}, y_{i}\right)$, for all $i<n$. 
A well-founded induction is terminal if its limit $\left(x_{n}, y_{n}\right)$ has no strict $A$-refinements.

A well-founded induction is an algebraical generalization of the well-founded model construction defined by Van Gelder et al. [1991]. The first type of refinement corresponds to making a partial structure more precise by applying Fitting's immediate consequence operator; the second type of refinement corresponds to making a structure more precise by eliminating an unfounded set. All terminal well-founded inductions of $A$ have the $A$-well-founded fixpoint as limit [Denecker and Vennekens, 2007].

When we introduce semantics for AIC based on AFT in the next section, we provide examples of the different constructions considered here.

\section{AFT-style Semantics for AICs}

A Semantic Operator for AICs Recall that in this paper, we only consider normal AICs.

Given a fixed database $D B$, the sets of update actions $\mathcal{U}$ that are of interest to us are those such that (i) $\mathcal{U}$ is consistent and (ii) each action in $\mathcal{U}$ modifies $D B$. As argued by Cruz-Filipe [2016], the set of such sets is isomorphic to $2^{A t}$. Hence, from now on, we identify such a set with a subset of $A t$ (the atoms whose value is changed by $\mathcal{U}$ ). If $a \in A t$ and $D B$ is a database, we define ch $a$ to be the update action $+a$ if $a \notin D B$ and $-a$ if $a \in D B$. Thus, in the above identification, a set of atoms $\overline{\mathcal{U}} \subseteq A t$ is identified with the set

$$
\mathcal{U}=\{\operatorname{ch} a \mid a \in \overline{\mathcal{U}}\}
$$

of update actions. Usually, we omit the bar, and simply write $\mathcal{U}$ for the subset of $A t$ as well.

Following the principle of minimality of change [Winslett, 1990; Eiter and Gottlob, 1992], we prefer smaller sets of updates. The lattice we are interested in is thus $\left\langle 2^{A t}, \subseteq\right\rangle$, where smaller elements correspond to "better" repairs.

Now consider a set of AICs $\eta$. Cruz-Filipe associated with such a set a semantic operator $\mathcal{T}_{\eta}: 2^{A t} \rightarrow 2^{A t}$ such that

$$
\mathcal{T}_{\eta}(\mathcal{U})=\mathcal{U} \biguplus\{\operatorname{head}(r) \mid r \in \eta \wedge \mathcal{U}(D B) \models \operatorname{body}(r)\} .
$$

Cruz-Filipe argued that a semantics for AICs based on grounded fixpoints of $\mathcal{T}_{\eta}$ coincides with the intuitions on a large set of examples and that it solves problems with several previously existing semantics. In the following paragraph, we define an approximator for $\mathcal{T}_{\eta}$ and hence, obtain a set of AFT-based semantics for AICs, based on similar intuitions.

An Approximator for $\mathcal{T}_{\eta}$ A partial action set is a mapping $\mathfrak{U}: A t \rightarrow\{\mathbf{t}, \mathbf{f}, \mathbf{u}\}$. A partial action set $\mathfrak{U}$ is two-valued if $\mathfrak{U}(A t) \subseteq\{\mathbf{t}, \mathbf{f}\}$; in this case, we identify $\mathfrak{U}$ with the action set $\{\operatorname{ch} a \mid \mathfrak{U}(a)=\mathbf{t}\}$. The intended reading of such a mapping is that $\mathfrak{U}(a)$ is true if $a$ is changed by $\mathfrak{U}$, it is false if $a$ is not changed by $\mathfrak{U}$ and it is unknown if $\mathfrak{U}$ leaves it open whether or not $a$ is changed. Alternatively, a partial action set is identified with an element of $\left(2^{A t}\right)^{c}$ (as standard). The set of all partial action sets is denoted $P$. The truth order $\leq_{t}$ on threevalued truth values is defined by $\mathbf{f} \leq_{t} \mathbf{u} \leq_{t} \mathbf{t}$. The inverse of a truth value is $\mathbf{f}^{-1}=\mathbf{t}, \mathbf{t}^{-1}=\mathbf{f}, \mathbf{u}^{-1}=\mathbf{u}$.
A partial database is a mapping $\mathfrak{D} \mathfrak{B}: A t \rightarrow\{\mathbf{t}, \mathbf{f}, \mathbf{u}\}$. The intended reading is that $\mathfrak{D} \mathfrak{B}(a)$ is true if $a$ is in the database, $\mathfrak{D} \mathfrak{B}(a)$ is false if $a$ is not in the database and $\mathfrak{D P}(a)$ is unknown otherwise.

If $\mathfrak{U}$ is a partial action set and $D B$ is a (regular) database, then we define $\mathfrak{U}(D B)$ to be the partial database such that

$$
\mathfrak{U}(D B): a \mapsto \begin{cases}D B(a) & \text { if } \mathfrak{U}(a)=\mathbf{f} \\ D B(a)^{-1} & \text { if } \mathfrak{U}(a)=\mathbf{t} \\ \mathbf{u} & \text { otherwise, }\end{cases}
$$

where $D B(a)=\mathbf{t}$ if $a \in D B$ and $D B(a)=\mathbf{f}$ otherwise.

Definition 3.1. Given a partial database $\mathfrak{D} \mathfrak{B}$, a set of AICs $\eta$ and an update action $\alpha$, we define the support of $\alpha$ with respect to $\langle\mathfrak{D} \mathfrak{B}, \eta\rangle$ as

$$
\operatorname{supp}_{\mathfrak{D} \mathfrak{B}, \eta}(\alpha)=\max _{\leq t}\left\{\operatorname{nup}(r)^{\mathfrak{D} \mathfrak{B}} \mid r \in \eta \wedge \operatorname{head}(r)=\alpha\right\},
$$

where $\operatorname{nup}(r)^{\mathfrak{D} \mathfrak{B}}$ refers to the standard three-valued truth evaluation of the formula ${ }^{1} \operatorname{nup}(r)$ in the partial interpretation $\mathfrak{D B}$ based on Kleene's truth tables [Kleene, 1938].

Using this notion, we define two additional values.

Definition 3.2. If $a \in A t, \mathfrak{U}$ is a partial set of update actions and $\langle D B, \eta\rangle$ as before, we define

$$
\begin{aligned}
\operatorname{supp}_{D B, \eta, \mathfrak{U}}^{c h}(a) & =\operatorname{supp}_{\mathfrak{U}(D B), \eta}(\operatorname{ch} a) \\
\operatorname{supp}_{D B, \eta, \mathfrak{U}}^{k e e p}(a) & =\operatorname{supp}_{\mathfrak{U}(D B), \eta}\left((\operatorname{ch} a)^{D}\right)
\end{aligned}
$$

As before, we often drop $D B$ and $\eta$ from the notation if they are clear from the context. Intuitively, $\operatorname{supp}_{\mathfrak{U}}^{c h}(a)$ is true if there is support for changing $a$ after updating the database with $\mathfrak{U}$, i.e., if at least one rule $r$ with head $(r)=\operatorname{ch} a$ has $\operatorname{nup}(r)^{\mathfrak{U}(D B)}=\mathbf{t}$; $\operatorname{supp}_{\mathfrak{U}}^{c h}(a)$ is unknown if it is not true and there is at least one rule $r$ with head $(r)=\operatorname{ch} a$ that has $\operatorname{nup}(r)^{\mathfrak{U}(D B)}=\mathbf{u}$; otherwise $\operatorname{supp}_{\mathfrak{U}}^{c h}(a)$ is false. Similarly, $\operatorname{supp}_{\mathfrak{U}}^{\text {keep }}(a)$ expresses the support for keeping $a$ as it is in the database (hence the name choice).

Example 3.3. Consider $D B=\emptyset$ and the following set $\eta$ :

$$
\neg a \wedge b \supset+a \quad a \wedge c \wedge d \supset-a .
$$

Consider $\mathfrak{U}=\{a \mapsto \mathbf{t}, b \mapsto \mathbf{t}, c \mapsto \mathbf{t}, d \mapsto \mathbf{u}\}$. Then $\operatorname{supp}_{D B, \eta, \mathfrak{U}}^{c h}(a)=\mathbf{t}$ and $\operatorname{supp}_{D B, \eta, \mathfrak{U}}^{k e e p}(a)=\mathbf{u}$.

Definition 3.4. Given $D B$ and $\eta$, we define an operator $\mathfrak{T}_{\langle D B, \eta\rangle}: P \rightarrow P$, as follows:

- If $\mathfrak{U}(a)=\mathbf{f}$, then $\mathfrak{T}_{\langle D B, \eta\rangle}(\mathfrak{U})(a)=\operatorname{supp}_{\mathfrak{U}}^{c h}(a)$.

- If $\mathfrak{U}(a)=\mathbf{t}$, then $\mathfrak{T}_{\langle D B, \eta\rangle}(\mathfrak{U})(a)=\operatorname{supp}_{\mathfrak{U}}^{k e e p}(a)^{-1}$.

- Otherwise (i.e., if $\mathfrak{U}(a)=\mathbf{u}$ ):

- if $\operatorname{supp}_{\mathfrak{U}}^{c h}(a)=\mathbf{t}$ and $\operatorname{supp}_{\mathfrak{U}}^{k e e p}(a)=\mathbf{f}$, then $\mathfrak{T}_{\langle D B, \eta\rangle}(\mathfrak{U})(a)=\mathbf{t}$;

- if $\operatorname{supp}_{\mathfrak{U}}^{\text {keep }}(a)=\mathbf{t}$ and $\operatorname{supp}_{\mathfrak{U}}^{c h}(a)=\mathbf{f}$, then $\mathfrak{T}_{\langle D B, \eta\rangle}(\mathfrak{U})(a)=\mathbf{f}$;

- otherwise, $\mathfrak{T}_{\langle D B, \eta\rangle}(\mathfrak{U})(a)=\mathbf{u}$.

When $D B$ is clear from the context, we write $\mathfrak{T}_{\eta}$ for $\mathfrak{T}_{\langle D B, \eta\rangle}$.

\footnotetext{
${ }^{1}$ Technically, $\operatorname{nup}(r)$ is a set of literal; we identify it with its conjunction here.
} 
Definition 3.4 is motivated as follows. Assume $\mathfrak{U}$ is a partial update set containing information on the intended update. In this case $\mathfrak{T}_{\eta}(\mathfrak{U})$ represents a revised update, using the AICs in $\eta$. In the case where $\mathfrak{U}(a)=\mathbf{f}, a$ is not an element of the (partial) update set at hand. The only way to add $a$ to the update is if some rule supports changing $a$, which is captured by supp ${ }^{c h}$. The case for $\mathfrak{U}(a)=\mathbf{t}$ is completely symmetrical, in this case the only reason for removing $a$ from the update at hand is if there is some rule that supports keeping $a$. In the last case, where $\mathfrak{U}(a)=\mathbf{u}$, we have no information what the update does to $a$ yet. In this case, we can derive that $a$ must be in the update if we already have support for ch $a$ and we are sure that there is no support for keeping $a$ (for $\operatorname{ch} a^{D}$ ). Similarly, we can derive that a must not be in the update if we have support for $\operatorname{ch} a^{D}$ but not for $\operatorname{ch} a$. In all other cases, we derive nothing about $a$ being in the update or not.

Proposition 3.5. $\mathfrak{T}_{\eta}$ is an approximator of $\mathcal{T}_{\eta}$.

Proof. (Sketch) The first step is to show that $\mathfrak{T}_{\eta}$ is $\leq_{p^{-}}$ monotone. Since Kleene-valuation is $\leq_{p}$-monotone, the functions that map $\mathfrak{U}$ to $\operatorname{supp}_{\mathfrak{U}}^{\text {ch }}(a)$ and $\operatorname{supp}_{\mathfrak{U}}^{\text {keep }}(a)$ for each $a \in A t$ are also $\leq_{p}$-monotone. Monotonicity of $\mathfrak{T}_{\eta}$ then follows from case analysis on the definition of $\mathfrak{T}_{\eta}(\mathfrak{U})(a)$.

The second step is to show that $\mathcal{T}_{\eta}$ and $\mathfrak{T}_{\eta}$ coincide on twovalued sets, which again can be established by case analysis on the definition of $\mathfrak{T}_{\eta}(\mathcal{U})(a)$.

Since $\mathfrak{T}_{\eta}$ is an approximator, it defines a family of semantics for AICs.

Definition 3.6. Let $\langle D B, \eta\rangle$ be a database.

- A partial stable repair of $\langle D B, \eta\rangle$ is a partial update set $\mathfrak{U}$ such that $\mathfrak{U}$ is a partial $\mathfrak{T}_{\eta}$-stable fixpoint. A stable repair is a partial stable repair that is two-valued.

- The AFT-well-founded repair of $\langle D B, \eta\rangle$ is the $\mathfrak{T}_{\eta}$-wellfounded fixpoint (in general, this is a partial update set).

- The Kripke-Kleene repair of $\langle D B, \eta\rangle$ is the $\mathfrak{T}_{\eta}$-KripkeKleene fixpoint (in general, this is a partial update set).

- A partial grounded repair of $\langle D B, \eta\rangle$ is a partial update set $\mathfrak{U}$ such that $\mathfrak{U}$ is a partial $\mathfrak{T}_{\eta}$-grounded fixpoint [Bogaerts et al., 2015b]. A grounded repair is a partial grounded repair that is two-valued.

The terminology in the above definition uses "repairs" for certain classes of fixpoints of a semantic operator. It follows easily that all two-valued update sets that are called "repair" in the the above definition, indeed are repairs according to AIC terminology. This paper is the first work that studies partial (non-two-valued) repairs.

The well-founded semantics induced by AFT can in general differ from the existing well-founded semantics for AICs, as we show in Example 3.13. To distinguish the two, we use the term AFT-well-founded semantics.

Grounded repairs were defined previously by Cruz-Filipe [2016]. All other classes of repairs defined in Definition 3.6 are newly introduced semantics by the current paper. We now illustrate these semantics by means of some examples.

Example 3.7. Consider the following set $\eta$ of AICs:

$$
\begin{array}{rrr}
\neg a \supset+a & \neg a \wedge \neg b \wedge \neg c \supset+c \\
a \wedge \neg b \supset+b & a \wedge c \wedge b \supset-b
\end{array}
$$

with $D B=\emptyset$. The $\mathfrak{T}_{\eta}$-well-founded fixpoint can be computed as the limit of the following well-founded induction:

$$
\begin{aligned}
& \mathfrak{U}_{0}: a \mapsto \mathbf{u}, b \mapsto \mathbf{u}, c \mapsto \mathbf{u} . \\
& \mathfrak{U}_{1}=\mathfrak{T}_{\eta}\left(\mathfrak{U}_{0}\right): a \mapsto \mathbf{t}, b \mapsto \mathbf{u}, c \mapsto \mathbf{u} \\
& \mathfrak{U}_{2}: a \mapsto \mathbf{t}, b \mapsto \mathbf{u}, c \mapsto \mathbf{f} \\
& \mathfrak{U}_{3}=\mathfrak{T}_{\eta}\left(\mathfrak{U}_{2}\right): a \mapsto \mathbf{t}, b \mapsto \mathbf{t}, c \mapsto \mathbf{f},
\end{aligned}
$$

where $\mathfrak{U}_{2}$ is an unfounded refinement of $\mathfrak{U}_{1}$ and all other refinements are application refinements. Note that unfoundedness refinements serve to minimize repairs.

Example 3.8. Consider the following set $\eta$ of AICs:

$$
\begin{array}{rlrl}
a & \wedge \neg b \supset+b & \neg a \wedge b \supset+a \\
\neg a \wedge \neg b \wedge \neg c \supset+c &
\end{array}
$$

with $D B=\emptyset$. Intuitively, we expect $+c$ to be an element of "good" repairs, and (following the minimality of change principle), no other actions to be in "good" repairs.

The $\mathfrak{T}_{\eta}$-well-founded fixpoint can be computed as the limit of the following well-founded induction:

$$
\begin{aligned}
\mathfrak{U}_{0}: a & \mapsto \mathbf{u}, b \mapsto \mathbf{u}, c \mapsto \mathbf{u} . \\
\mathfrak{U}_{1}: a & \mapsto \mathbf{f}, b \mapsto \mathbf{f}, c \mapsto \mathbf{u} . \\
\mathfrak{U}_{2}=\mathfrak{T}_{\eta}\left(\mathfrak{U}_{1}\right): a & \mapsto \mathbf{f}, b \mapsto \mathbf{f}, c \mapsto \mathbf{t} .
\end{aligned}
$$

Here, the first refinement is an unfoundedness refinement. In can be verified that $\mathfrak{U}_{0}$ is a fixpoint of $\mathfrak{T}_{\eta}$ and hence is the Kripke-Kleene repair. Again, the AFT-well-founded repair is two-valued and corresponds to the intuitions.

As can be expected, not every set of AICs has a two-valued well-founded repair. That would simply be too much to ask. It would mean that for every set of AICs we can unambiguously identify a single repair. The following example illustrates that this is indeed not always the case. It also illustrates that (for this specific example), $\mathfrak{T}_{\eta}$-stable repairs provide a solution that corresponds to the intuitions.

Example 3.9. Consider the following set $\eta$ of AICs:

$$
\begin{array}{rlrl}
\neg a & \wedge \neg b \supset+a & \neg a \wedge \neg b \supset+b \\
a \wedge \neg c \supset+c &
\end{array}
$$

with $D B=\emptyset$. Intuitively, $\eta$ has two "good" repairs. The first two rules state that $a$ or $b$ should be added in order to "fix" the violated constraint $\neg(\neg a \wedge \neg b)$. Depending on that choice, the last rule determines whether or not $c$ should be repaired. The two intended repairs are thus $\{+a,+c\}$ and $\{+b\}$. In can be verified that these two intended repairs are indeed stable and that the AFT-well-founded repair maps all atoms to $\mathbf{u}$ (hence it approximates the two intended repairs).

Properties of AFT-Style Semantics We now show that all of these semantics are nicely invariant under shifting.

Proposition 3.10. $\mathfrak{T}_{\eta}$, and hence also $\mathcal{T}_{\eta}$, is invariant under shifting, i.e., for each set $S \subseteq$ At:

$$
\mathfrak{T}_{\langle D B, \eta\rangle}=\mathfrak{T}_{\left\langle\operatorname{shift}_{S}(D B), \operatorname{shift}_{S}(\eta)\right\rangle}
$$

Corollary 3.11. All AFT-style semantics for AICs have the shifting property. 
Proposition 3.12. If the AFT-well-founded repair is twovalued, it is also well-founded (as defined by Cruz-Filipe et al. [2013]).

Example 3.13. The converse of Proposition 3.12 does not hold. Consider for instance the following set $\eta$ of AICs (based on Example 18 of Cruz-Filipe [2016])

$$
\begin{array}{rlr}
\neg a & \supset+a & \neg a \wedge \neg b \supset+b \\
\neg b \wedge \neg c \supset+c &
\end{array}
$$

and $D B=\emptyset$. In this case, the AFT-well-founded repair is $\mathcal{U}_{A F T}: a \mapsto \mathbf{t}, b \mapsto \mathbf{f}, c \mapsto \mathbf{t}$. This is clearly the intended repair. Indeed, the first rule dictates that $a$ needs to be changed (here: added) no matter what. The second rule then becomes void (there is never a reason to add $b$ ) and the last rule dictates that also $c$ must be added. However, $a \mapsto \mathbf{t}, b \mapsto \mathbf{t}, c \mapsto \mathbf{f}$ is also a well-founded repair, obtained by first applying the second rule and then the first one.

Proposition 3.14. All $\mathfrak{T}_{\eta}$-stable repairs are justified.

Example 3.15. The converse of Proposition 3.14 does not hold. Consider the following set $\eta$ of AICs.

$$
\begin{array}{rlr}
\neg a & \supset+a & a \wedge \neg b \supset+b \\
a \wedge \neg b & \supset-a
\end{array}
$$

with $D B=\emptyset$. In this case $\{+a,+b\}$ is a justified repair of $\langle D B, \eta\rangle$, but not a stable repair. To see that it is not a stable repair, recall that we identify a partial action set with an element of $\left(2^{A t}\right)^{c}$, e.g., $\{a \mapsto \mathbf{u}, b \mapsto \mathbf{u}\}$ is identified with $(\emptyset,\{a, b\})$. It now suffices to note that $\mathfrak{T}_{\eta}(\emptyset,\{a, b\})=$ $(\emptyset,\{a, b\})$ and hence $\operatorname{lfp} \mathfrak{T}_{\eta}(\cdot,\{a, b\})_{1}=\emptyset \neq\{a, b\}$.

To see that it is a justified repair, note that $\{+a,+b\}$ is the least set closed under $\eta$.

While the converse of Proposition 3.14 does not hold in general, for a broad class of active integrity constraints, it does hold. We first define this class and then prove that this is indeed the case.

Definition 3.16. A set of AICs $\eta$ is called unipolar if there are no rules $r, r^{\prime} \in \eta$ with head $(r)=\operatorname{head}\left(r^{\prime}\right)^{D}$.

Unipolar AICs make sense in practice, for example if there are tables from which removing data is never an option.

Proposition 3.17. If $\eta$ is unipolar, then each justified repair of $\langle D B, \eta\rangle$ is $\mathfrak{T}_{\eta}$-stable.

Using Lemma 19 of Cruz-Filipe [2016], which states that all justified repairs are grounded, we easily find how justified repairs and the AFT-well-founded repair relate.

Corollary 3.18. The AFT-well-founded repair and the Kripke-Kleene repair approximate all justified repairs.

\section{Complexity Analysis}

We begin this section by stating an observation about the complexity of computing $\mathfrak{T}_{\eta}$. All complexity results are in terms of the size of the database, $\langle D B, \eta\rangle$.

Proposition 4.1. $\mathfrak{T}_{\eta}$ is computable in polynomial time.

As a consequence, we get the following complexity results.
Proposition 4.2. The Kripke-Kleene repair for $\langle D B, \eta\rangle$ is computable in polynomial time.

Proposition 4.3. The ATF-well-founded repair for $\langle D B, \eta\rangle$ is computable in polynomial time.

Proposition 4.4. The task of checking if a database $\langle D B, \eta\rangle$ has a stable repair is NP-complete.

For each of our semantics, complexity is the same as the complexity of its counterpart in (normal) logic programming. This illustrates that the added expressivity (allowing AICs that are not unipolar) does not result in added complexity.

\section{Conclusion}

In this paper, we defined an approximator in the domain of active integrity constraints. The result is a family of semantics for AICs based on existing intuitions in various domains of non-monotonic reasoning. We studied properties of our induced semantics. In particular the AFT-well-founded semantics possesses desirable properties: it approximates all repairs of various families (stable, justified, grounded) and hence can be used for approximate skeptical query-answering with respect to any of these semantics. Furthermore, the AFT-wellfounded repair can be computed in time polynomial in the size of the database.

Our study is far from finished. In the context of approximation fixpoint theory, ultimate approximators have been studied by Denecker et al. [2004]. They showed that with each two-valued operator, we can associate a canonical approximator. The ultimate approximator induces another family of semantics for AICs. In other domains, e.g., in logic programming, semantics based on ultimate approximators have some very desirable properties, but in general come at the cost of a higher computational complexity than their "standard" variants. It remains to be researched if the same holds in the context of AICs. In this paper, we showed that the class of justified repairs is situated in between the classes of stable and of grounded repairs. It is known from AFT that the class of ultimate stable fixpoints also falls in between the classes of stable fixpoints (for any approximator) and grounded fixpoints. Hence, an interesting research question would be to verify if justified repairs coincide with ultimate stable fixpoints in this domain, and if not, how they relate. Another topic with potential for interesting future work are inconsistencies. Consider for instance the set of AICs $\{\neg a \supset+a, a \supset-a\}$; intuitively, we expect a semantic operator to derive an inconsistency from any partial action set; in standard AFT this is not possible. However, extensions of AFT that accommodate this have been defined [Bi et al., 2014]; it would be interesting to see how AICs fit in this general theory. Another AFT-based topic of interest could be to study what safe inductions [Bogaerts et al., 2017] yield in the context of AICs and whether they can fix problems with the well-founded semantics. One last topic on which more extensive research might be needed is the domain of revision programming [Marek and Truszczynski, 1998]. Caroprese and Truszczyński [2011] showed structural correspondences between semantics for AICs and semantics for revision programs. Our paper now paves the way to applying AFT to revision programming as well. 


\section{References}

[Abiteboul, 1988] Serge Abiteboul. Updates, a new frontier. In Marc Gyssens, Jan Paredaens, and Dirk van Gucht, editors, ICDT, volume 326 of $L N C S$, pages 1-18. Springer, 1988.

[Bi et al., 2014] Yi Bi, Jia-Huai You, and Zhiyong Feng. A generalization of approximation fixpoint theory and application. In Proceedings of $R R$, pages 45-59, 2014.

[Bogaerts et al., 2015a] Bart Bogaerts, Joost Vennekens, and Marc Denecker. Grounded fixpoints and their applications in knowledge representation. AIJ, 224:51-71, 2015.

[Bogaerts et al., 2015b] Bart Bogaerts, Joost Vennekens, and Marc Denecker. Partial grounded fixpoints. In Proceedings of IJCAI, pages 2784-2790, 2015.

[Bogaerts et al., 2016] Bart Bogaerts, Joost Vennekens, and Marc Denecker. On well-founded set-inductions and locally monotone operators. ACM Trans. Comput. Logic, 17(4):27:1-27:32, September 2016 .

[Bogaerts et al., 2017] Bart Bogaerts, Joost Vennekens, and Marc Denecker. Safe inductions: An algebraic study. In Proceedings of IJCAI, 2017. (to appear).

[Brewka and Woltran, 2010] Gerhard Brewka and Stefan Woltran. Abstract dialectical frameworks. In Proceedings of $K R$, pages 102-111, 2010.

[Caroprese and Truszczyński, 2011] Luciano Caroprese and Mirosław Truszczyński. Active integrity constraints and revision programming. TPLP, 11(6):905-952, 2011.

[Caroprese et al., 2006] Luciano Caroprese, Sergio Greco, Cristina Sirangelo, and Ester Zumpano. Declarative semantics of production rules for integrity maintenance. In Proceedings of ICLP, pages 26-40, 2006.

[Cruz-Filipe et al., 2013] Luís Cruz-Filipe, Graça Gaspar, Patrícia Engrácia, and Isabel Nunes. Computing repairs from active integrity constraints. In TASE 2013, pages 183-190. IEEE Computer Society, 2013.

[Cruz-Filipe et al., 2015] Luís Cruz-Filipe, Michael Franz, Artavazd Hakhverdyan, Marta Ludovico, Isabel Nunes, and Peter Schneider-Kamp. repAIrC: A tool for ensuring data consistency by means of active integrity constraints. In Proceedings of KMIS, volume 3, pages 17-26, 2015.

[Cruz-Filipe, 2014] Luís Cruz-Filipe. Optimizing computation of repairs from active integrity constraints. In Proceedings of FoIKS, pages 361-380, 2014.

[Cruz-Filipe, 2016] Luís Cruz-Filipe. Grounded fixpoints and active integrity constraints. In Technical communications of ICLP, pages 11.1-11.14, 2016.

[Denecker and Vennekens, 2007] Marc Denecker and Joost Vennekens. Well-founded semantics and the algebraic theory of non-monotone inductive definitions. In Proceedings of LPNMR, pages 84-96, 2007.

[Denecker et al., 2000] Marc Denecker, Victor Marek, and Mirosław Truszczyński. Approximations, stable operators, wellfounded fixpoints and applications in nonmonotonic reasoning. In Logic-Based Artificial Intelligence, Springer, volume 597, pages 127-144, 2000.

[Denecker et al., 2003] Marc Denecker, Victor Marek, and Mirosław Truszczyński. Uniform semantic treatment of default and autoepistemic logics. AIJ, 143(1):79-122, 2003.
[Denecker et al., 2004] Marc Denecker, Victor Marek, and Mirosław Truszczyński. Ultimate approximation and its application in nonmonotonic knowledge representation systems. Information and Computation, 192(1):84-121, July 2004.

[Dung, 1995] Phan Minh Dung. On the acceptability of arguments and its fundamental role in nonmonotonic reasoning, logic programming and n-person games. AI, 77(2):321 - 357, 1995.

[Eiter and Gottlob, 1992] Thomas Eiter and Georg Gottlob. On the complexity of propositional knowledge base revision, updates, and counterfactuals. AIJ, 57(2-3):227-270, 1992.

[Flesca et al., 2004] Sergio Flesca, Sergio Greco, and Ester Zumpano. Active integrity constraints. In Proceedings of SIGPLAN, pages 98-107, 2004.

[Kleene, 1938] Stephen Cole Kleene. On notation for ordinal numbers. The Journal of Symbolic Logic, 3(4):150-155, 1938.

[Marek and Truszczynski, 1998] V. Wiktor Marek and Miroslaw Truszczynski. Revision programming. Theor. Comput. Sci., 190(2):241-277, 1998.

[Moore, 1985] Robert C. Moore. Semantical considerations on nonmonotonic logic. AIJ, 25(1):75-94, 1985.

[Przymusinski and Turner, 1997] Teodor C. Przymusinski and Hudson Turner. Update by means of inference rules. Journal of Logic Programming, 30(2):125-143, 1997.

[Reiter, 1980] Raymond Reiter. A logic for default reasoning. AIJ, 13(1-2):81-132, 1980.

[Strass, 2013] Hannes Strass. Approximating operators and semantics for abstract dialectical frameworks. AIJ, 205:39-70, 2013.

[Teniente and Olivé, 1995] Ernest Teniente and Antoni Olivé. Updating knowledge bases while maintaining their consistency. VLDB Journal, 4(2):193-241, 1995.

[van Emden and Kowalski, 1976] Maarten H. van Emden and Robert A. Kowalski. The semantics of predicate logic as a programming language. J. ACM, 23(4):733-742, 1976.

[Van Gelder et al., 1991] Allen Van Gelder, Kenneth A. Ross, and John S. Schlipf. The well-founded semantics for general logic programs. J. ACM, 38(3):620-650, 1991.

[Vennekens et al., 2006] Joost Vennekens, David Gilis, and Marc Denecker. Splitting an operator: Algebraic modularity results for logics with fixpoint semantics. ACM Trans. Comput. Log., 7(4):765-797, 2006.

[Vennekens et al., 2007a] Joost Vennekens, Maarten Mariën, Johan Wittocx, and Marc Denecker. Predicate introduction for logics with a fixpoint semantics. Part I: Logic programming. Fundamenta Informaticae, 79(1-2):187-208, September 2007.

[Vennekens et al., 2007b] Joost Vennekens, Maarten Mariën, Johan Wittocx, and Marc Denecker. Predicate introduction for logics with a fixpoint semantics. Part II: Autoepistemic logic. Fundamenta Informaticae, 79(1-2):209-227, September 2007.

[Widom and Ceri, 1996] Jennifer Widom and Stefano Ceri, editors. Active Database Systems: Triggers and Rules For Advanced Database Processing. Morgan Kaufmann, 1996.

[Winslett, 1990] Marianne Winslett. Updating Logical Databases. Cambridge Tracts in Theoretical Computer Science. Cambridge University Press, 1990. 